\title{
The Research of Taxi Resource Configuration
}

\author{
Zhuo Zhang \\ School of the North China Electric Power University, Baoding 071000, China. \\ zz605243615@qq.com
}

\begin{abstract}
This paper mainly studies the Internet age taxi resource allocation problems, we establish the synthetic evaluation model between supply and demand by look into the data. For the degree of the matching of supply and demand "in the different time and space, you will first need to query a large amount of data. This article grabbed drops quickly in intelligent travel platform a taxi in Beijing taxi quantity distribution and demand quantity, on this basis, the zoning model was established. We divide Beijing into ten regions of (see figure 1). Then we confirm the supply and demand ratio, waiting time, empty rate of the three indicators, and establish the model of fuzzy comprehensive evaluation based on AHP. Through the principle of analytic hierarchy process, we determine the index weight of indexes in different areas of the comprehensive evaluation on the same day, get different regions of the degree of the matching of supply and demand. The result shows A, C, E, J area matching degree between supply and demand for good, area F for good, D, G, H, I area for the poor.
\end{abstract}

Keywords: Division; the AHP and fuzzy comprehensive evaluation.

\section{1. 数据准备}

查找数据是本题的关键。通过查找, 我们得到滴滴快的智能出行平台的实时数据。我们 选取最有代表性的北京, 由于数据量较大，我们用 CooSeeker 进行数据抓取，选取 9 月 6 日 的数据进行分析（6日为工作日）（见材料 1)。

\section{2. 区域划分模型}

\section{1 模型的建立}

北京市城区面积较大，为了方便讨论，我们将北京市城区划分为 $\mathrm{n}$ 份，对每个区域中出 租车总量和需求人数进行讨论。运用编程进行求解, 得出 0 时至 23 时每个区域出租车总量和 需求人数, 利用 Matlab 做图像曲线分析。

\section{2 模型的求解}

运用 Matlab 编程进行划分, 综合考虑北京市交通状况, 对数据进行笁选, 我们、以经度 116.27、116.43, 纬度 39.9、39.8 为分界线将其划分为 10 个区域较为合理（见图 1），求 和代表整个区域整体水平（见表 1)。通过查找资料发现, 安装有滴滴快的的出租车占北京 总出租车 $90.3 \%^{[1]}$; 据中国电子商务研究中心监测数据显示, 滴滴快的使用率为 $10.23 \%{ }^{[2]}$, 我 们近似认为用软件打车的人数占总需求人数 $10.23 \%$, 利用这两个数据求得 9 个区域 0 到 $23 \mathrm{~h}$ 出租车总量及总需求人数。（见表 2)

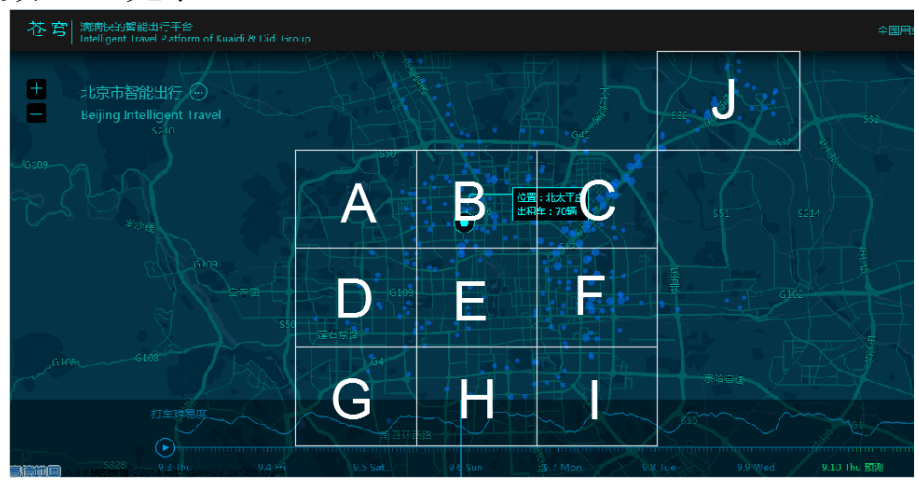

图 1 区域划分示意图 


\section{3. 基于AHP_模糊综合评价 ${ }^{[3]}$}

\section{1 指标建立与分析}

应对供求匹配程度分析, 我们建立三项指标, 分别为需供比, 等车时间和空驶率。需供 比通过需求与出租车供给相比得到, 等车时间和空驶率通过查询出租车 OD 分析平台得到。

表 1 需供比

\begin{tabular}{|c|c|c|c|c|c|c|c|c|c|c|}
\hline \multicolumn{11}{|c|}{ 需供比 } \\
\hline & A & B & $\mathrm{C}$ & D & $\mathrm{E}$ & $\mathrm{F}$ & $\mathrm{G}$ & $\mathrm{H}$ & I & $\mathrm{J}$ \\
\hline 1.00 & inf & 0.41 & 1.03 & 1 & 3.23 & 1.44 & 1 & inf & inf & 0.09 \\
\hline 2.00 & inf & 0.10 & 0.39 & 0.00 & 0.33 & 0.11 & 1 & inf & 1 & 0.05 \\
\hline 3.00 & $\inf$ & 0.10 & 0.12 & inf & 0.23 & 0.20 & 1 & 3.60 & $\inf$ & 0.09 \\
\hline 4.00 & 1 & 0.14 & 0.14 & 0.00 & 0.22 & 0.19 & 1 & inf & 1 & 0.00 \\
\hline 5.00 & inf & 0.24 & 0.23 & 0.00 & 0.43 & 0.14 & 0.00 & 1.17 & 1 & 0.03 \\
\hline 6.00 & 4.01 & 0.58 & 0.46 & 0.00 & 0.71 & 0.67 & 5.30 & inf & inf & 0.06 \\
\hline 7.00 & $\inf$ & 0.80 & 0.51 & 0.38 & 0.64 & 1.93 & 8.25 & inf & inf & 0.09 \\
\hline 8.00 & 2.57 & 1.43 & 0.82 & 2.81 & 0.88 & 6.89 & inf & 10.25 & 7.31 & 0.06 \\
\hline 9.00 & 0.66 & 2.25 & 1.08 & 1.27 & 1.57 & 7.02 & 2.21 & 2.39 & 11.11 & 0.13 \\
\hline 10.00 & 0.83 & 0.63 & 1.32 & 2.39 & 0.70 & 3.28 & 2.77 & 4.47 & 3.51 & 0.07 \\
\hline 11.00 & 0.80 & 0.34 & 0.59 & inf & 0.58 & 3.70 & inf & $\inf$ & $\inf$ & 0.10 \\
\hline 12.00 & 8.42 & 0.28 & 0.37 & inf & 0.36 & 3.70 & inf & inf & inf & 0.18 \\
\hline 13.00 & 2.26 & 0.26 & 0.26 & inf & 0.54 & 1.43 & inf & inf & inf & 0.07 \\
\hline 14.00 & 2.79 & 0.33 & 0.57 & inf & 0.56 & 4.54 & inf & inf & inf & 0.19 \\
\hline 15.00 & 1.42 & 0.42 & 0.75 & inf & 0.99 & 2.53 & inf & inf & 3.53 & 0.20 \\
\hline 16.00 & 0.67 & 0.41 & 0.73 & 2.51 & 0.90 & 4.02 & inf & $\inf$ & inf & 0.26 \\
\hline 17.00 & 1.61 & 0.35 & 0.62 & inf & 1.41 & 2.39 & inf & inf & inf & 0.14 \\
\hline 18.00 & 1.55 & 0.57 & 0.80 & inf & 1.59 & 3.81 & inf & inf & inf & 0.21 \\
\hline 19.00 & 0.65 & 1.15 & 2.79 & inf & 3.10 & 7.91 & inf & 9.71 & 2.21 & 0.52 \\
\hline 20.00 & 1.35 & 0.60 & 3.36 & inf & 1.96 & 10.23 & inf & inf & inf & 0.85 \\
\hline 21.00 & 0.59 & 0.49 & 2.17 & 1 & 1.69 & 3.29 & inf & inf & 7.22 & 1.53 \\
\hline 22.00 & 1.45 & 0.76 & 3.76 & inf & 0.44 & 2.54 & inf & inf & inf & 0.65 \\
\hline 23.00 & 2.09 & 0.96 & 2.16 & inf & 1.40 & 0.81 & inf & inf & 3.39 & 1.06 \\
\hline 24.00 & 1.17 & 0.51 & 1.17 & inf & 1.36 & 0.50 & inf & inf & inf & 0.22 \\
\hline
\end{tabular}

3.2 模型原理

(1)层次分析法的原理

层次分析法通过建立层次分析模型，将有关各因素按不同属性分解为若干层次，并将每 一层次的各因素进行两两比较, 得到对比矩阵, 计算对比矩阵的最大特征值及特征向量, 从 而得到下一层次相对于上一层次的重要程度，进而得到权重。

(2)模糊综合评价原理

将待考察的模糊概念或模糊对象作为一定的模糊集合，建立适当的隶属函数，从而对模 糊对象进行定量分析。

3.3 模型的求解

(1)根据比较尺度理论，构造两两比较阵 $\mathrm{A}$ :

$$
\begin{aligned}
& A=\left(a_{i j}\right)_{n \times n}(i, j=1,2, \ldots, n) \\
& a_{i j}=1 / a_{j i}
\end{aligned}
$$

我们根据经验进行打分, 得比较矩阵:

$$
R=\left[\begin{array}{ccc}
1 & 3 / 4 & 5 / 8 \\
4 / 3 & 1 & 5 / 6 \\
8 / 5 & 6 / 5 & 1
\end{array}\right]
$$


其中, $a_{11}$ 为供需比/供需比, $a_{12}$ 为等待时间/供需比, $a_{13}$ 为空驶率/供需比, $a_{22}$ 为等待 时间/等待时间, $a_{33}$ 为空驶率/空驶率。

(2)判断矩阵 $\mathrm{A}$ 对应于最大特征值 $\lambda_{\text {max }}$ 的特征向量 $\mathrm{W}$ ，经归一化后即为同一层次相应因素 对于上一层次某因素相对重要性的排序权值。由于在综合全部比较结果时难免包含一定程度 的非一致性, 因此需要对其进行一致性检验,

计算一致性指标 $C I$ :

$C I=\frac{\lambda_{\max }-n}{n-1}$

根据查找的 $R I(0.58)$ 计算一致性比例:

$C R=\frac{C I}{R I}$

当 $C R \leqslant 0.10$ 时, 认为比较阵的一致性是可以接受的, 否则应对比较阵进行适当修改。

运用 Mat lab 计算得 $C R=0$, 认为一致性可接受。

(3)确定评价对象评价指标 $C$

$C=\left(c_{1}, c_{2}, c_{3}\right)$

$c_{1}$ 为供需比, $c_{2}$ 为等待时间, $c_{3}$ 为空驶率。

(4)确定评价集 $V$

$V=\left(v_{1}, v_{2}, v_{3}, v_{4}, v_{5}\right)$

$v_{1}$ 为优秀, $v_{2}$ 为良好, $v_{3}$ 为一般, $v_{4}$ 为较差, $v_{5}$ 差。

(5)建立隶属度矩阵 $T$ 。在构造了等级模糊子集后, 要逐个对被评事物从每个因素上进行 量化, 即确定从单因素来看被评事物对等级模糊子集的隶属度, 从而得到隶属度矩阵 $T$ 。我 们依据经验确定评价级, 确定每项指标对优秀、良好、一般、较差、差的隶属度, 得到隶属 度矩阵。

66定评价因素的权向量 $W$ 。在模糊综合评价中, 使用层次分析法确定评价因素的权向量:

$W=\left(w_{1}, w_{2}, w_{3}\right)$

$w_{1}$ 为供需比占权重, $w_{2}$ 为等待时间占比, $w_{3}$ 为空驶率所占比重。

利用 Matlab 进行求解得:

$w_{1}=0.4068$

$w_{2}=0.3390$

$w_{3}=0.2542$

(7)合成模糊综合评价结果矩阵 $S$ 。采用 “加权平均型” 模型, 原理是利用合适的算子将 $W$ 与各被评事物的 $T$ 进行合成, 得到各被评事物的模糊综合评价结果向量从而对效益进行评价。 即:

$S=W * T$

利用 Matlab 进行计算得到评价结果矩阵 


$$
S=\left[\begin{array}{rrrrr}
0.3277 & 0.2104 & 0.1165 & 0.0925 & 0.2528 \\
0.4386 & 0.2796 & 0.1398 & 0.0614 & 0.0805 \\
0.4555 & 0.2458 & 0.1059 & 0.1292 & 0.0635 \\
0.2175 & 0.1094 & 0.0763 & 0.0756 & 0.5212 \\
0.4336 & 0.2083 & 0.25 & 0.0445 & 0.0635 \\
0.2429 & 0.2528 & 0.1278 & 0.2112 & 0.1653 \\
0.1631 & 0.1412 & 0.0706 & 0.0445 & 0.5805 \\
0.0636 & 0.1582 & 0.0318 & 0.0869 & 0.6596 \\
0.1462 & 0.1518 & 0.0494 & 0.1596 & 0.493 \\
0.4047 & 0.1292 & 0.1208 & 0.0953 & 0.25
\end{array}\right]
$$

\section{4. 结论}

取数值最大的评语作为综合评判结果 (见表 3)

表 3 综合评判结果

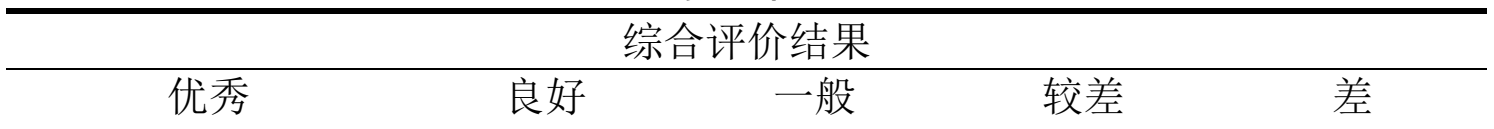

A、B、C、E、J F G、H、I

我们得到的评判结果较为可靠, 仅有 B 区和 J 区定性分析结果有一定差距, 我们得到较 好的评判指标。

\section{References}

[1] Taxi speed road network, 2013-2014 software market analysis report, http://www.sfw.cn/xinwen/439655.html, 2015/9/11.

[2] Beijing traffic development research center, 2006 Beijing traffic development of the annual report. http://wenku.baidu.com/link?url=lurBMIQpmV9f_v6FwGk0N_0pzd178TzBZVH_dE rQ2IFve878gciNKiLpuKhHY4nYqs7GYUTiy82gaY96EqPP6D4C_8_ved05TOjFhqytTa,2015/ $9 / 12$.

[3] Juliang Jin, Yiming Wei, Ding Jing etc. The fuzzy comprehensive evaluation model based on improved analytic hierarchy process [J]. Journal of water conservancy, 2004, (3) : 65-65. The DOI: $10.3321 /$ j.i SSN: 0559-9350.2004.03.011. 